\title{
Vital Rates from the Action of Mutation Accumulation
}

\author{
Kenneth W. Wachter • David R. Steinsaltz • \\ Steven N. Evans
}

Received: 15 August 2008 / Accepted: 11 November 2009 / Published online: 1 January 2010 (C) The Author(s) 2009. This article is published with open access at Springerlink.com

\begin{abstract}
New models for evolutionary processes of mutation accumulation allow hypotheses about the age-specificity of mutational effects to be translated into predictions of heterogeneous population hazard functions. We apply these models to questions in the biodemography of longevity, including proposed explanations of Gompertz hazards and mortality plateaus.
\end{abstract}

Keywords Evolution • Genetic load • Senescence • Gompertz hazard • Mortality plateau

\section{Mutation Accumulation}

Why are flies, worms, and humans subject to laws of age-specific adult mortality that are uncannily similar in shape? After suitable species-specific changes in scale, organisms with different environments, life histories, body

\footnotetext{
K. W. Wachter

Department of Demography, University of California, Berkeley, 2232 Piedmont Avenue, Berkeley, CA 94720-2120, USA

e-mail:wachter@demog.berkeley.edu

D. R. Steinsaltz $(\bowtie)$

Department of Statistics, University of Oxford, 1 South Parks Road, Oxford, OX1 3TG, UK

e-mail: steinsal@stats.ox.ac.uk

S. N. Evans

Department of Statistics, University of California, Berkeley, 367 Evans Hall, Berkeley, CA 94720-3860, USA

e-mail: evans@stat.berkeley.edu
} 
plans, and lifespans turn out to resemble each other in the statistics of their demise. Similarities are typically expressed in terms of hazard functions. The hazard function is a summary measure of rates of death by age across a population, equal to the negative slope of the logarithm of the population survivorship function. Hazard functions for populations from many species show two common features: exponential increase with age over a stretch of ages and attenuated increase over later ages, generating the visual appearance of a plateau. The recognition of these commonalities goes back at least as far as Pearl and Miner (1935); the generalisation and quantitative elaboration have been signal achievements of the new biodemography, summed up by Vaupel et al. (1998), Carey (2003), Wachter and Finch (1997), Carey and Tuljapurkar (2003).

Explanations for shared features of senescent mortality across species are sought in considerations from reliability engineering, from optimal lifehistory theory, and from evolutionary processes of antagonistic pleiotropy and mutation accumulation. Reliability engineering is a functional approach to senescence, picturing the organism as a machine with some component structure, attempting to derive the failure modes of the whole from some presumably simpler failure modes of the components. The aim is usually to draw inferences from qualitative classes of structures to general shapes of mortality curves. The enterprise is considered successful if the broad features common to many real-world mortality rates are reproduced in the model. Some examples are Strehler and Mildvan (1960), Rosen (1978), Gavrilov (1978), Gavrilov and Gavrilova (1991), Weitz and Fraser (2001), Finkelstein and Esaulova (2006).

Functional models start from the structure of the organism, while evolutionary models pose prior questions: What kind of machine is the organism, and why is it put together the way it is? Many functional models lead to the same general pattern for mortality rates, after all, and each generic class of models can yield diverse shapes of age-specific mortality. Optimal life-history approaches try to narrow down the choices a priori, by explaining why a given structural framework, or a certain choice of parameters within the structural framework, might be evolutionarily preferred. Much work in this area (for example, Rose 1985; Schnebel and Grossfield 1988; Hedrick 1999; Wachter 1999; Williams and Day 2003) builds on the concept of antagonistic pleiotropy, introduced into the theory of senescence by George Williams (1957). Williams held that early reproduction and late survival would be negatively associated through direct genetic mechanisms. Recent research often abstracts from the genetic term "pleiotropy", to contemplation of more general trade-offs and compromises that operate across time within the lifetime of an organism and across generations (cf. Toupance et al. 1998; Hasty 2001; Campisi 2003; Nyström 2003).

The other side of the conventional evolutionary theory of aging, called mutation accumulation, views senescence not as an optimal trade-off between early- and late-life reproductive success, but rather as the age-specific effect of genetic load, a concept developed by Peter Medawar (1952). Ongoing random 
mutation spews mostly deleterious changes into the genome. Since the only genetic "repair mechanism" is the death of the organism carrying the defect, there is perpetually an overhang of deaths not yet realized, stretching from the time of the initial mutation until all descendants have died from the effect of the allele. Less nocive mutations linger. Since an individual may not live long enough to experience the harm from a late-acting mutation, this provides another process through which natural selection reshapes demographic schedules. At equilibrium, mortality rates trend upward with age in proportion to the weakening force of selection. The population observed at any given time will be found to be genetically heterogeneous, because new mutations with particular age effects are scattered independently across the individuals in a population and the mutations act together to alter each individual's internal susceptibilities to causes of death.

All these approaches have something to contribute to an understanding of the central phenomenon at issue, that risks of impairment and death increase with age. None of the approaches excludes the others. This article treats mutation accumulation, but in a way that incorporates, as a start, one characteristic feature from reliability models, early-age concomitants of lateage debilitation. In future work we hope to tackle head-on the challenge of linking evolutionary models with mechanistic and physiological models. Trade-offs and impacts on age-specific mortality must be embodied in complex reliability structures. With the exception of Pletcher and Neuhauser (2000), the mathematical development on both sides has up to now lacked the flexibility required for a more synoptic model.

Decades of research have established, piece by piece, a mathematical framework for characterizing genetic load and the interplay between mutation, selection, and recombination. Developments through the end of the Twentieth Century are presented in an authoritative book by Bürger (2000). Early achievements addressed single-locus and several-locus systems with rich genetic structure, but did not attempt to superimpose demographic dimensions. During the 1990s, Brian Charlesworth (1994) succeeded in consolidating an age-specific demographic treatment based on a linear approximation. Charlesworth (2001) showed that both of the tell-tale common features of hazard functions across species, the exponential Gompertzian rise and the eventual onset of plateaus, could be predicted by the linear approximate model from simple, minimalist assumptions. His ideas have attracted wide attention.

Three obstacles have hitherto blocked the path to a broader application of mutation-accumulation models: first, the limited versions of age-specific genetic harm under consideration, second, the assumption that genetic loci affecting different ranges of ages evolve independently, and, third, inattention to heterogeneity.

The early work of W. D. Hamilton (1966) posited mutations that apply a single bolus of mortality at one fixed age, what we call a "point-mass" model. B. Charlesworth (2001) tried other stylized patterns: mortality increments within specified windows, in Gaussian shapes around specified centers, or beyond specified ages of onset. He also tried coupling these age-specific patterns with 
an increment independent of age; our coupling of late-age with early age effects follows in this spirit precedent.

A provision that late-acting effects carry with them some early manifestations is characteristic of reliability models for senescent mortality. A typical example is the model of Gavrilov and Gavrilova (2001), which posits an underlying structure of independent components and identifies death with the first component failure. Waiting time until death may have a mean or mode late in life, but its distribution will have a left-hand tail showing up in some early deaths. We appropriate this feature for our applications of mutation accumulation. Building evolutionary structure directly into reliability models remains a project for the future.

On the issue of independence, it is an essential feature of demographicallybased models that the evolution at distinct sites fails to be independent even if sites act independently; that is, even if the mortality increment due to two alleles co-occurring is merely the sum of their individual effects. To put it simply, death comes to an individual only once, so that any mutation that increases mortality makes a second mutation that also increases mortality less costly, as measured in lost reproductive opportunity. Linearization, as previously employed, treats multiple mutations as though they were evolving independently and so misses the critical interaction effect in the cumulative demographic impact.

On the issue of heterogeneity, natural selection must have variability on which to act. Selection can only balance mutation when some members of the population carry more deleterious mutant alleles than others. The levels of the mean counts of mutant alleles at equilibrium are altered by the variability of counts about their means, the variability which drives the whole mutation-selection process. The feedback from variances to means is typically suppressed by linearization.

All three of these imperatives, flexible profiles for effects, interactions, and heterogeneity, call for a fully nonlinear model, such as the one applied here. The need for such a model seems to have been appreciated already in Brian Charlesworth's (2001) pathbreaking paper. In his Section 4 he sought to incorporate nonlinear interactions through an iterative numerical procedure, making survivorship at each step in time depend on the previous mean accumulation of mutant alleles en route to an equilibrium. This procedure suppresses heterogeneity and leads to different answers from our fully nonlinear model, but in some circumstances it generates usable approximations. It highlights the importance of nonlinear effects. Even in the "point-mass" setting, nonlinearity can produce outcomes qualitatively different from those predicted with the linearized approach, as shown in Steinsaltz et al. (2005) and Wachter et al. (2008). The full model also makes it possible to prove conditions for the existence of equilibria and Walls of Death.

In our application here, mutant alleles arise that each increase age-specific mortality rates according to the profile of a Gamma probability density function. The model builds in the nonlinear demographic interactions among the 
accumulating mutant alleles, and takes explicit account of the heterogeneity in genetic endowment among individuals. Investigating a range of choices of parameter values, we show that the features of prime demographic interest, Gompertzian stretches and late-age plateaus, can be produced within this setting.

The Gamma profiles adopted here are reminiscent of functional forms common in reliability models, but no precise analogy is intended. Our choice was guided by the idea that the "essential organs" of Gavrilov and Gavrilova (1991) might be replaced by a large number of "useful organs", of similar internal redundancy, whose propensity to failure could be triggered or exacerbated by the presence of one or more mutant alleles. Ultimately we hope it will be feasible to situate reliability models explicitly within the context of mutation accumulation. The evolutionary unified failure theory of our aspiration would also need to incorporate elements of optimal life history, as well as accounting for the complex hierarchy of trade-offs, from the level of single genes and organelles up to ecosystems, and on timescales from the milliseconds of RNA transcription to the millennia of evolutionary time.

For our present, more modest, purposes the Gamma family was chosen because it has the desired property that late-age increments in mortality are systematically tied to early-age increments in a fashion that varies smoothly with the mean age of effect. This specification takes us beyond the highly stylized setting of point-mass cases, while retaining enough familiarity for ready interpretation.

We describe the model in the section headed "The Mutation-Selection Model", the formulas that go into demographic calculations in the section headed "Formulas", and the detailed specification of ingredients and parameters in the section headed "Specifications". We present the mortality outcomes predicted by the theory in the section headed "Predictions".

\section{The Mutation-Selection Model}

Medawar's idea of mutation accumulation as a cause of senescence depends upon the action of large numbers of mutations, each with small deleterious effects on survival at specific ranges of age. Mutations which affect young ages are weeded out of the population quickly by natural selection, because members who carry them contribute fewer offspring to the next generation. Mutations affecting older individuals, with less reproductive potential remaining to lose, are weeded out less rapidly. While weeding progresses, new mutations are being introduced at random into the population. Mutant alleles accumulate until a balance is reached between the force of mutation and the force of selection. All things being equal, the less costly mutations-those that produce their harm later-will be more common at equilibrium.

Our model for mutation accumulation is an infinite-population model in continuous time with large or infinite numbers of genetic loci, in the tradition 
of a famous paper by Kimura and Maruyama (1966). The model comes in two versions. The version applied here incorporates what we call "Free Recombination", in which recombination is assumed to operate on a more rapid time scale than mutation and selection. For mathematical details we refer the reader to Wachter et al. (2008) and to Evans et al. (2009) which shows that this version can be regarded as a limiting case of discrete-generation models in the limit of weak selection and mutation. A companion version in which recombination is assumed to be negligible is developed in Steinsaltz et al. (2005). Our two treatments of recombination bracket a potential continuum of more complex treatments.

For each version of the model, there are analytic solutions available to describe entire time trajectories for the population. In this paper, we are primarily concerned with equilibrium states. Equilibrium states are distributions of genotypes which are stable in time under the joint action of mutation and selection. In many situations, including those treated here, we can prove that there is a unique equilibrium state, and that this state represents the distribution to which the population converges over time.

The accumulating mutations under study here are germ-line mutations maintained in the genome over long stretches of evolutionary time. Our framework may also have some application to somatic mutations accumulating within the cells of an individual individual organism during its lifecourse, but that is not our current focus.

The model has three ingredients which must be specified for each application. First is a set of profiles for the age-specific action of deleterious mutant alleles. Second is a specification of the rates at which mutant alleles of different kinds arise, the mutation part of mutation-selection balance. Third is a function determining selective cost, the selection part of mutation-selection balance. In this section we develop the framework, with notation and formulas in the following section. Our choices for the ingredients, which serve as illustrations in this section, are spelled out in detail in the section headed "Specifications".

Our first ingredient is a set of profiles for age-specific action. Examples of profiles are the four functions of age in Fig. 1, a figure discussed further in the section headed "Specifications". A profile is added onto the age-specific hazard function for each mutant allele carried by an individual.

In general, we posit a set $\mathcal{M}$ of potential mutations fitted with a geometric structure to allow us to describe the process of picking a new set of random mutations which are passed on to the next generation. In our application, the profiles form a one-parameter family of curves, and we can identify $\mathcal{M}$ with the interval of the real line containing the permitted values of the parameter. Picking a set of random mutations comes down to picking a random set of points from the real line, what probability theorists call a point process, in this case a Poisson point process. The important feature of the profiles is their dependence on age. No attempt is being made to identify alleles with genes on chromosomes or otherwise to model biological structures. Versatile agespecific structure is achieved in conjunction with a degree of stylization in the representation of the genome. 
Fig. 1 Gamma profiles for increments to the hazard function for four selected values of the mutation index $m$, namely $1.125,2.250,4.125$, and 6.000 (from left to right as they rise from the axis). Background parameters are $\alpha=15$ and $\phi=1 / 20$, and $\eta=0.100$

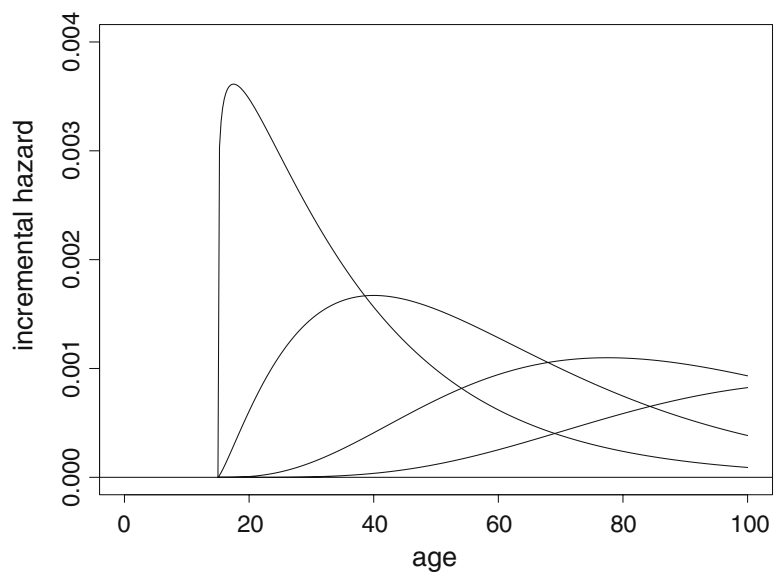

Our second ingredient gives rates at which mutant alleles arise, rates expressed in general by a measure $v$ on $\mathcal{M}$. When we take $\mathcal{M}$ to be a real interval, we can identify $v$ with a non-negative function, the density of the measure, and we often take $v$ constant over the interval for the sake of having a neutral choice. Time is continuous in our model. It is convenient to scale the time axis so that one unit of time corresponds to one generation in discrete settings. The rate $v$ is then expressed in units of mutations per generation.

Our third ingredient is a selective cost function $S$. It evaluates (on a logarithmic scale) the loss in fitness produced by any batch of mutant alleles which an individual may carry. The alleles are dominant, back-mutation is not allowed, and costs are evaluated under the assumption that age-specific fertility rates $f_{x}$ are being rescaled to keep population size stationary and are otherwise exogenous. The general form of the model allows fertility as well as mortality to be shaped endogenously by the action of deleterious mutations with a background level of any chosen form, but those options are not pursued in this paper. In the present context, as discussed in Wachter et al. (2008), following Charlesworth (2000), page 930, there are good reasons for identifying the selective cost of a batch of mutations with the resulting lifetime loss of net reproduction, and we do so here.

\section{Formulas}

We now introduce formal notation and describe how the ingredients of our model fit together in terms of the formulas from which predictions are calculated.

We use the letter $g$ and the word "genotype" as shorthand to refer to the finite batch of mutant alleles carried by a member of the population. Alleles with the same profile of action are treated as copies of the same allele even 
though they are found at different sites in the genome. An individual without mutant alleles has the "null genotype" $g=0$ with wild-type alleles at every site.

The survivorship function $\ell_{x}(g)$ for a subpopulation of members with genotype $g$ is the proportion of members of the subpopulation living beyond age $x$. When we take the logarithm of $\ell_{x}(g)$ and multiply by -1 , we obtain the cumulative hazard function, whose derivative, when it exists, is the hazard function itself, also called the "force of mortality". The cumulative hazard function at age $x$ is the area under the hazard function up to age $x$. We work with cumulative hazard functions in order to have simpler expressions for survivorships $\ell_{x}$, as well as to have formulas that apply without change to discrete-age and continuous-age cases.

We write $\eta(m) \kappa(m, x)$ for the increment to the cumulative hazard function at age $x$ produced by allele $m$ from $\mathcal{M}$. The cumulative profile function $\kappa(m, x)$ - the area up to age $x$ under a curve like the curves in Fig. 1represents the shape of the age profile of mortality effects, normalised to have total effect 1: that is, with $\int_{0}^{\infty} \kappa(m, x) d x=1$. The factor $\eta(m)$ then adjusts the overall size of the effect.

To write the survivorship function, we start with an exogenous baseline cumulative hazard $\Lambda(x)$ and add to it a term $\eta(m) \kappa(m, x)$ for each $m$ in the batch $g$ to obtain the cumulative hazard. We multiply the cumulative hazard by -1 and apply the exponential function to obtain the survivorship.

We write capital $G$ for the random batch of mutant alleles carried by an individual selected at random from the population. The count of alleles in $G$ with values of $m$ in any given subset of $\mathcal{M}$ is thus a random variable. An example would be the count of alleles with parameters between 2 and 3. The mean of this random variable is just the population average number of mutant alleles in the interval $(2,3)$, and it equals the area within the interval $(2,3)$ under a curve $\rho$ called the intensity of $G$.

We now touch on some probability theory, the tool which enables us to go beyond linear approximations and treat interactions and heterogeneity. The intensity $\rho$ gives information about overall genetic load, but on its own it may not provide a complete description of the genetics of the population. The function $\rho$ only specifies for each region of $\mathcal{M}$ the population average number of mutant alleles in that region and a priori does not enable one to compute the proportion of the population that have more than some number of mutant alleles in a given region of $\mathcal{M}$ or to determine whether a randomly chosen individual who happens to have larger than expected numbers of mutant alleles in one region is more or less likely to have a larger than expected number in another region. In this model, there is a distribution of genotypes, which are batches of mutant alleles from $\mathcal{M}$; the intensity only describes the overall frequency of each mutation, with no information about its genetic partners.

When selective costs are linear-effectively, the non-epistatic case in which distinct loci evolve independently - the genotype distribution is a Poisson random measure, a mathematical construct whose properties are described, for instance, in Kallenberg (1983). Intuitively, the genotype of an individual sampled at random from the population can be described by going through $\mathcal{M}$ 
point by point, and taking mutations independently at random with probabilities governed by $\rho$. In the setting of interest to us, however, when the selective cost is nonlinear, there will be a complex structure of interactions between mutations.

It is surprising, then, that the simple Poisson structure returns, regardless of the complexity of the epistasis, in our model with Free Recombination, as shown in Evans et al. (2009). While distinct loci now do not evolve independently, the distribution at any given time does have a the structure of a Poisson random measure and is completely described by the intensity alone. The population average or "expectation value" of any quantity of interest depends only on $\rho$; we use the notation $\mathbb{E}_{\rho}$.

Our goal, then, is to determine the intensity $\rho(m)$ of mutations at equilibrium. From it we can find the expected (aggregate) population survival curve $\mathbb{E}_{\rho}\left[\ell_{x}(G)\right]$, the proportion of the whole population living beyond age $x$.

The selective cost $S(g)$ for genotype $g$ is calculated under our assumption of zero population growth and is given by the difference in its Net Reproduction Ratio from the Net Reproduction Ratio for the null genotype:

$$
S(g):=\int f_{x} \ell_{x}(0) d x-\int f_{x} \ell_{x}(g) d x .
$$

Survivorship for genotype $g$ is given by

$$
\ell_{x}(g)=\exp \left(-\Lambda(x)-\sum_{m \in g} \eta(m) \kappa(m, x)\right) .
$$

Section 3 in Wachter et al. (2008) shows that the general formulas in Evans et al. (2009) along with properties of Poisson point processes imply that aggregate survivorship is given by

$$
\mathbb{E}_{\rho}\left[\ell_{x}(G)\right]=\ell_{x}(0) \exp \left(-\int\left(1-e^{-\eta \kappa(m, x)}\right) \rho(m) d m\right) .
$$

The slope of minus the logarithm of the left-hand side is the population hazard. The increment to the cumulative population hazard due to the accumulation of copies of allele $m$ can be written

$$
H(m, x)=\left(1-e^{-\eta \kappa(m, x)}\right) \rho(m) .
$$

In this way the left-hand side of Eq. 3 takes the form $\ell_{x}(0) \exp \left(-\int H(m, x) d m\right)$. The equilibrium intensity $\rho$ has to satisfy

$$
0=v(m)-\rho(m) \int\left(1-e^{-\eta \kappa(m, x)}\right) f_{x} \mathbb{E}_{\rho}\left[\ell_{x}(G)\right] d x
$$


The Eq. 5 can be solved numerically by an iterative scheme. We start out with $\rho_{0} \equiv 0$, corresponding to the null genotype and, supposing we have already constructed the approximate solutions $\rho^{0}, \ldots, \rho^{n}$, define $\rho^{n+1}$ by

$$
\rho^{n+1}(m):=v(m) /\left[\int\left(1-e^{-\eta \kappa(m, x)}\right) f_{x} \mathbb{E}_{\rho^{n}}\left[\ell_{x}(G)\right] d x\right] .
$$

Under appropriate conditions, it is possible to prove that this sequence does in fact converge to a solution of Eq. 5. For our numerical calculations, we approximate the continuous range of values of $m$ by a grid with one thousand points and evaluate integrals over age by a grid with steps of 0.10 years. Calculations are implemented in the open-source R Statistical System based on the computer system $\mathrm{S}$ developed at Bell Laboratories.

\section{Specifications}

We now turn to the detailed specification of the cases treated in this paper, going into the particular choices for the three ingredients of the model and accompanying parameters.

The first ingredient, the profiles for mutational action, have been introduced in the section headed "Mutation Accumulation". We set

$$
\kappa(m, x)= \begin{cases}(1 / \Gamma(m)) \int_{\alpha}^{x} \phi^{m}(y-\alpha)^{m-1} e^{-\phi(y-\alpha)} d y, & x \geq \alpha, \\ 0, & x<\alpha .\end{cases}
$$

The profile function $\kappa(m, x)$ is the cumulative distribution function for a shifted Gamma probability distribution. The Gamma shape parameter equals the index value $m$ and varies from allele to allele. The Gamma rate parameter $\phi$ is the same for all alleles. The shift $\alpha$ for the origin is the age of maturity; alleles affect only adult mortality. The quantity $\Gamma(m)$ is the ordinary gamma function. Each effect is assigned an effect size $\eta(m)$ which adjusts the strength of the action.

In addition to their association with reliability models, Gamma distribution functions offer advantages of familiarity and flexibility. They offer a clear contrast to the point-mass profiles going back to W. D. Hamilton already studied in Wachter et al. (2008). In the point-mass setting, $\kappa(m, x)$ is a unit step-function and $m$ indexes the age at the step. In our present setting, higher values of $m$ still correspond to later-acting alleles, but effects are spread across ages, with wider spread for later-acting alleles. Even late-acting alleles have some small effect at young adult ages, a salient difference from the point-mass case. The more the mutational effect is spread over older ages, the lower is the selective cost, and the more copies there will be of $m$ on average when natural selection manages to balance recurrent mutation. 
The mean age of action for allele $m$ is the mean of the shifted Gamma distribution $\alpha+m / \phi$, the mode is $\alpha+(m-1) / \phi$, and the standard deviation in age of action is $\sqrt{m} / \phi$.

Figure 1 shows the shapes of the age-specific increments to the hazard function for four typical alleles in our setting, with $\eta \equiv 0.100, \alpha=15$, and $m$ equal to $1.125,2.250,4.125$, and 6.000 . The value $\eta=0.100$ is a typical standard value. We discuss the impact of other values in the section headed "Predictions".

Our second ingredient, the mutation rate $v(m)$, is taken to be constant over an interval $[1, \xi]$ and zero outside it. In the choice of a constant mutation rate we follow the practice of Charlesworth (2001), seeking to keep our assumption about mutation as neutral as possible. The total rate per generation $v_{t o t}$ of the deleterious mutations treated in the model amounts to the length of the interval, $\xi-1$, times the $v$ value in the interval. We use $v_{t o t}$ as a label. It is the mean number of new mutations per zygote per generation. We consider cases with $\xi$ between 5 and 7 and $v_{t o t}$ between 0.120 and 0.170 per generation.

The final ingredient of model specification is the selective cost of a batch of mutations. We assume that mutations affect an individual's fitness only through their effect on mortality rates, and that the cumulative mortality effects of multiple mutations are additive contributions to the hazard function. Our selective cost function is a difference in Net Reproduction Ratios, quantities which depend on fertility as well as survival. We assume a fixed fertility schedule $f_{x}$ equal to 0 below an age of maturity $\alpha$ and above a latest age at reproduction $\beta$ and equal to a non-zero constant between these ages. The value of the constant is tuned to produce an overall stationary population size. For the predictions of the section headed "Predictions", $\alpha=15$ and $\beta=50$.

The inclusion of an upper age limit on fertility is important to the interpretation of our results. The values of the age-specific profile $\kappa(m, x)$ for $x$ above $\beta$ are irrelevant to the selective cost imposed by $m$ and therefore to the equilibrium frequency of $m$, but they make significant contributions to the predicted post-reproductive hazard. It is the association between early-age and late-age hazards built into our family of profiles that drives the predicted outcomes. Biologically, we are assuming a correlation between young and old ages in phenotypic effects. Reasons for doing so, in relation to reliability models for aging, have been discussed in the section headed "Mutation Accumulation". The correlation across ages prevents the occurrence of a Wall of Death at the end of reproduction, and shapes the old-age hazards.

The selective cost function also depends on the choice of baseline survival schedule, the schedule for the null genotype. Following the lead of Charlesworth (2001), we assume a constant baseline hazard $\lambda$ above the age of maturity $\alpha$, corresponding to a cumulative baseline hazard $\Lambda(x)=\lambda(x-\alpha)$ above $\alpha$ and zero below. Since we are rescaling fertility to achieve stationarity, pre-reproductive mortality can be ignored. The baseline hazard can be taken to represent a minimum realizable rate, sometimes identified with the socalled the extrinsic mortality rate despite the problems inherent in this notion discussed by Williams and Day (2003). As with fertility, our choice of baseline 
hazard is intended to be as neutral as possible, in order to concentrate on structure arising from the dynamics of mutation and selection.

\section{Predictions}

We now examine predicted hazard functions at mutation-selection equilibrium when the age-specific action of mutant alleles takes the form of Gamma profiles described in the section headed "Specifications". We begin with a case chosen to serve as a standard example, to which we shall compare other cases. It is illustrated in Fig. 2.

For our standard example, we set the upper cutoff on shape parameters $\xi=$ 6 , and the total mutation rate $v_{\text {tot }}=0.150$, along with an effect size $\eta$ constant at 0.100 . a baseline mortality level $\lambda=1 / 20$, and a rate parameter $\phi=1 / 20$. The maximum increment at any one age associated with our Gamma profiles is then a little more than three per thousand per year. For the sake of analogy with human life history, we set the age of initial reproduction (also the age of earliest action of the mortality profiles) to be $\alpha=15$, and the age at end of reproduction to be $\beta=50$.

Figure 2 shows the population hazard rate calculated from Eq. 3. It rises slowly from the background level and then accelerates, giving the impression of a Gompertz-Makeham curve in the middle of the age range, and straightening out at older ages. About one in ten-thousand individuals survive beyond age 70 .

In this illustration, the equilibrium density of mutations $\rho$ turns out to be closely approximated by an exponential function of the shape parameter, namely $\rho(m) \approx 0.170 \exp (1.377 \mathrm{~m})$. On average, individuals in the population carry about two mutant alleles with $m<2.0$, a bit over a dozen with $3.5<m<$ 4.0, and nearly three-hundred with $5.5<m<6.0$, for an average total of 526 .

Fig. 2 Predicted hazard for a standard example with $\lambda=\phi=1 / 20, v_{\text {tot }}=0.150$, $\xi=6, \alpha=15$, and $\beta=50$

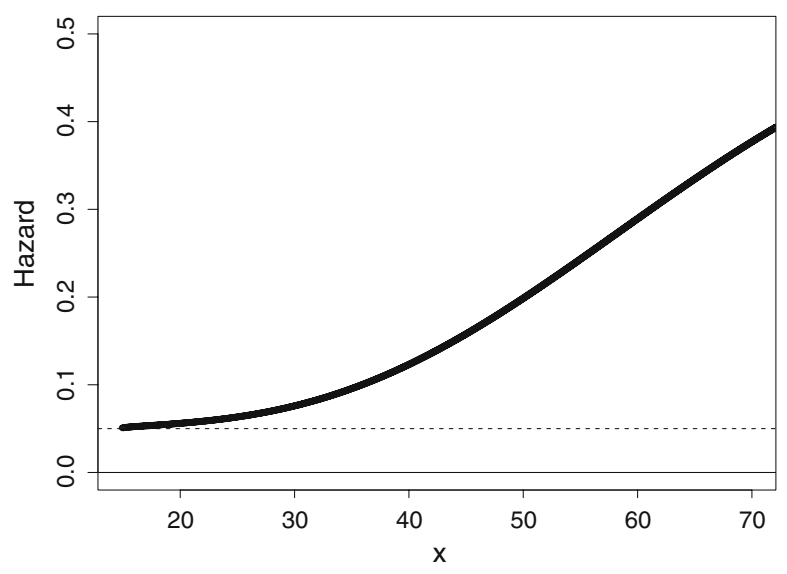


The Poisson standard deviation of the total number of alleles across individuals is around 23.

The effect for a given $m$ peaks at age $15+(m-1) / \phi$. The effects for $m<2.0$ are peaking before age 35 , an age to which most members of the population survive. The cost in net reproduction from an additional mutation affecting these ages is high, and selection keeps their equilibrium representation low. Effects for $m>5.5$ only become substantial at ages at which most individuals have already died. Selective costs are low and copies persist long enough to be found at high numbers in the population despite the rarity of new mutations.

Figure 3 shows the logarithm of the population hazard rate for three comparative cases. The standard example is the solid curve. The dotted curve has $v_{t o t}=0.170$ and an upper shape parameter cutoff of $\xi=5.5$. The dashed curve has $v_{t o t}=0.120$ and $\xi=7$. These alternatives have been chosen from among cases for which the predicted equilibrium hazard is between 0.300 and 0.550 at the age to which one in ten thousand survive, respectively equal to $69.9,71.9$, and 66.7 years.

The higher hazards at old ages in the dotted curve are due to the presence of later-acting alleles with $m$ ranging up to 7 . These alleles have effects whose age-specific profiles increase throughout the range of ages to which population members survive. The mode for $m=7$ is not reached until 135 years. Although the mutation rate is lower for the dotted curve, the shapes of the age-specific effects lead to higher hazards toward the end of life.

We see that mutation accumulation with the given profiles and parameters produces a long middle stretch of nearly loglinear hazards, corresponding to a Gompertz form. At young ages the curves are convex on the logarithmic scale, bending upward, as effects of mutant alleles come into play. At older ages, the curves turn concave. Accumulation of mutational effects concentrated at late ages is held in check by their small accompanying effects at young ages in this specification.

Fig. 3 Logarithm of predicted hazard for three cases showing early upward bend, straight middle Gompertzian stretch, and late downward bend. The cases all have $\lambda=\phi=1 / 20$ and $\eta \equiv 0.100$. The solid curve has $v_{\text {tot }}=0.150$, and $\xi=6.0$; the dashed curve has $v_{\text {tot }}=0.170$, and $\xi=5.5$; the dotted curve has $v_{\text {tot }}=0.120$, and $\xi=7.0$

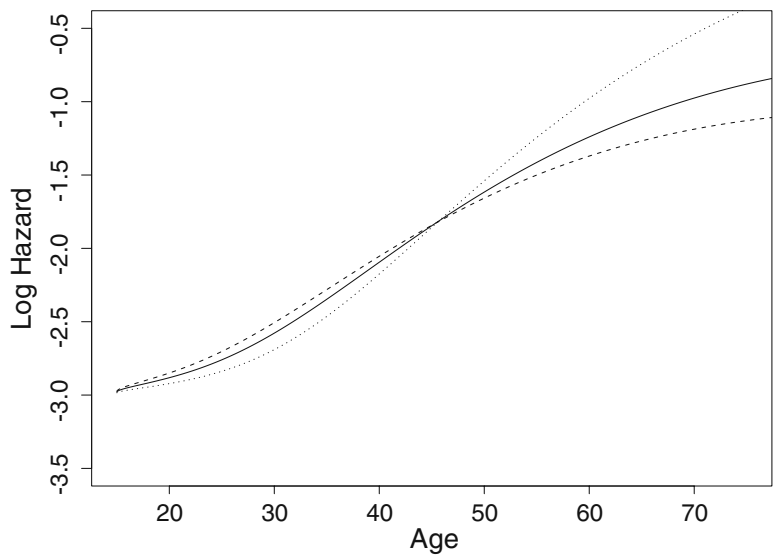


Fig. 4 Probability of survival by age for the standard example as predicted by the full nonlinear model (circles), by a linear approximate model (dots), and by the baseline model

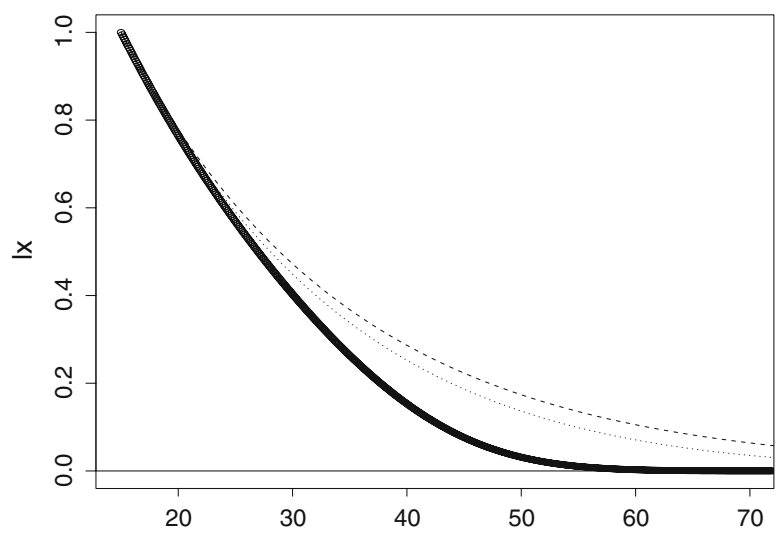

The interactions among effects at different ages taken into account by the nonlinear model turn out to have a substantial impact on predictions, as expected from results in Wachter et al. (2008). We compare predictions from the full nonlinear model to predictions from a linear approximate model of the kind on which earlier studies have relied. Figure 4 shows the population survivorship function for our standard example in a thick line, along with baseline survivorship in a dashed line, and survivorship from the linear approximate model between them in a dotted line. Only about a third of the reduction in life expectancy from 35.0 years to 28.8 years due to mutation accumulation is captured by the linear approximate model.

The sizes of effects, in contrast to their shapes, turn out to have only modest influence on the predictions. Alleles with smaller effects accumulate at equilibrium in greater numbers. Changes in the intensity $\rho$ roughly balance changes in effect size $\eta$. Figure 5 shows the predicted hazard functions with parameters taken from our standard example but with different choices of $\eta$.

Fig. 5 The influence of effect size is shown with predicted hazard functions from seven cases described in the text, some with indistinguishable outcomes, sharing all parameter values except effect size with the example of Fig. 1

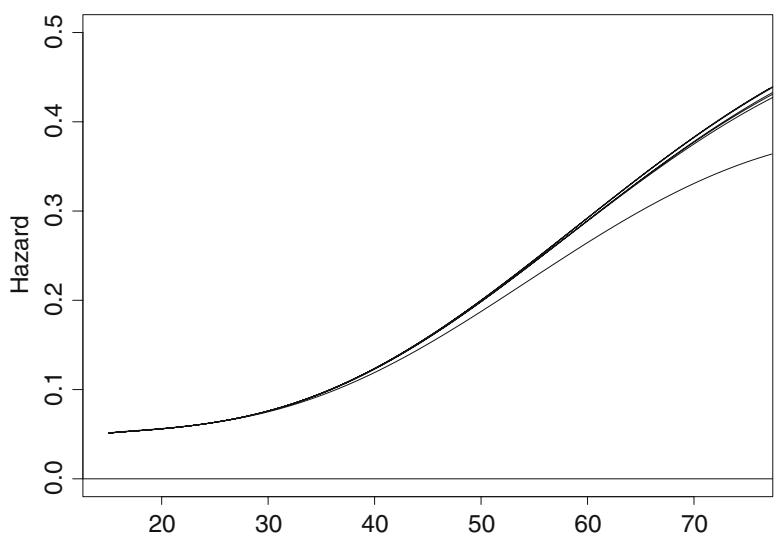


Three uppermost curves, almost indistinguishable, have constant $\eta=$ $0.0001, \eta=0.001$, and $\eta=0.010$. The mean total number of mutant alleles runs to a bit over five hundred thousand in the first case, fifty-thousand in the second, and five thousand in the third. Three slightly lower curves, also hardly distinguishable, include our example with $\eta$ constant at 0.100 and two examples with changing $\eta$, one rising linearly with the shape parameter from 0.020 to 0.200 and one falling linearly from 0.200 to 0.020 . Mean counts of alleles are 527, 309 and 1493 respectively. Small effect sizes accompany larger mean numbers when they occur for alleles with late action. The lowermost curve has $\eta=1.000$ and a mean of only 51 alleles. Only as $\eta$ becomes this large, outside the range of intended application of the model, do we see substantially different predicted hazard functions.

The approximate invariance of predicted hazard functions with effect sizes is an expression of Haldane's Principle, enunciated by Haldane (1937) and discussed in terms of our nonlinear models in Wachter et al. (2008). In Eq. 4, the contribution $H(m, x)$ from allele $m$ is nearly linear in $\eta(m) \rho(m)$ for small $\eta$, so scaling $\eta(m)$ up can be nearly compensated, allele by allele, by scaling $\rho(m)$.

One of the most familiar general predictions of the evolutionary theory of senescence is a positive relationship between the extrinsic mortality rate associated with unavoidable risks in natural settings such as predation and accidents that are present even the young and healthy, and the "rate of senescence" measured by the slope of the logarithm of the hazard rate with respect to age. Our predictions hint at such a relationship, but only for substantial values of the baseline hazard $\lambda$. Figure 6 shows the logarithms of the predicted equilibrium hazard for our standard set of parameters as the level of the constant baseline hazard is raised from 0.020 to 0.050 and on to 0.080 .

Slopes computed over the middle range of ages from 30 to 50 to which a Gompertz fit is roughly appropriate hover around 0.050 for the first two cases but rise to 0.074 as $\lambda$ increases to 0.080 . In cases not shown here in which non-

Fig. 6 The influence of the level of extrinsic hazards on the pace of senescent mortality is shown by predicted log hazards from three cases sharing parameters with the standard example of Fig. 1 except for $\lambda$, which ranges, from bottom to top, over values $0.020,0.050$, and 0.080

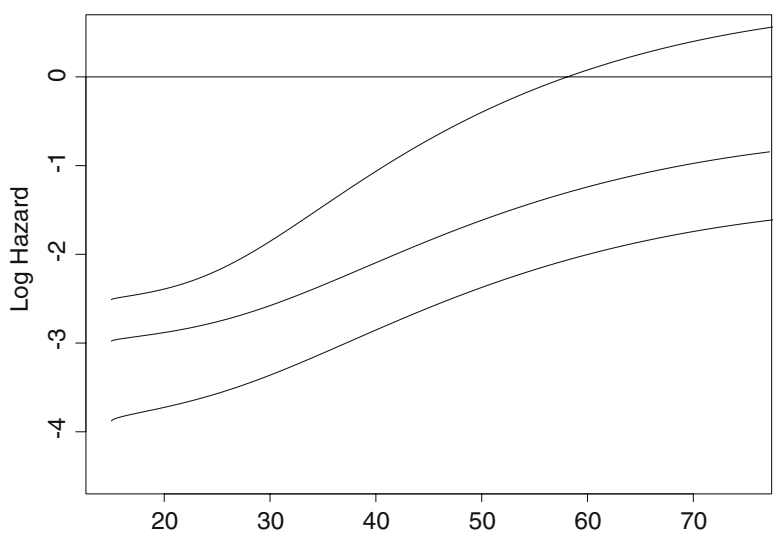


zero fertility extends to higher ages, there is a closer match between values of the slope and values of the parameter $\lambda$ itself, paralleling a relationship found with linear approximate models in Charlesworth (2001).

The stretch of ages with exponentially increasing hazards, corresponding to linear increase in log hazards, visible in Figs. 2 and 3 does not extend out to extreme ages. Attenuation of increase is already visible in the upper ages toward right of those figures. We focus on this attenuation in Fig. 7, which shows the predicted hazard rate for the same standard example of Fig. 2 but with a horizontal axis extending all the way out to an age of 120 years. The vertical scale also differs from Fig. 2. Around 100 years, the hazard levels off, establishing a brief plateau phase, and by 120 years a declining hazard is apparent. Only one in ten billion survive to 100 years with our standard parameter choices, but the plateau and subsequent decline are to be expected with parameters leading to milder mortality regimes as well.

The plateau at extreme ages is due to the property of the profiles for the age-specific action of mutant alleles to which we have already called attention, namely that even alleles whose action is spread over old ages all have some small effects at young ages. These small effects rein in the accumulation of late-acting mutant alleles. They prevent any Wall of Death; that is, any finite age at which the hazard rate goes to infinity and survivorship reaches zero. Walls of Death occur in many elementary cases for profiles with Hamiltonstyle, point-mass profiles, as shown in Wachter et al. (2008). The proposal for generating plateaus by assuming some small effects at young ages for all mutant alleles was put forward by Charlesworth (2001) and shown to be valid for the linear approximate model. We now see that these outcomes also hold in the full nonlinear model with the particular profiles we are studying.

In summary, we have found that the process of mutation accumulation can readily produce predicted population hazard functions with the chief features highlighted by the cross-species comparisons of biodemographers. It can produce a stretch of ages with an exponential, Gompertzian rise in hazards and it can produce a late-age hazard plateau. These outcomes arise from a set of

Fig. 7 A plateau in the predicted hazard function at extreme ages in the standard example of Fig. 2 with a longer range of ages

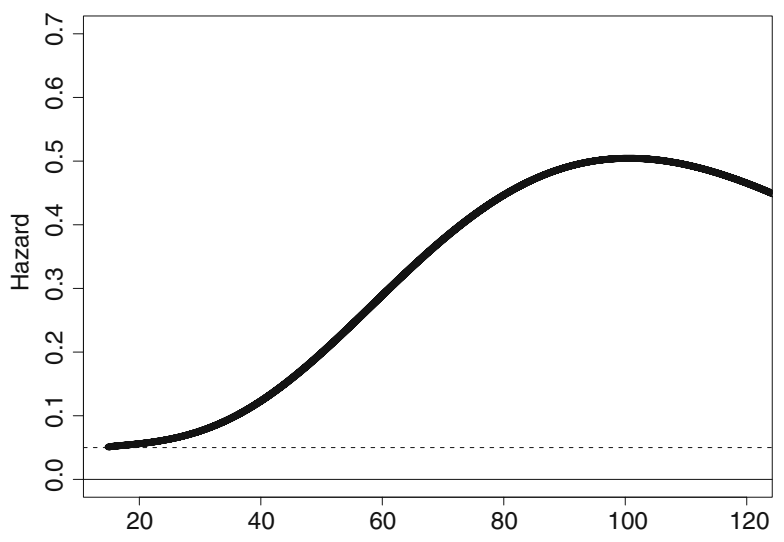


assumptions about the age-specific action of mutant alleles that are suggested by examples from reliability theory and from the functional approach to the study of senescence. It remains, however, to develop comprehensive models in which the generic mutation accumulation machinery is driven by plausible genetic and physiological mechanisms, and in which age-specific tradeoffs are derived compellingly from reliability theory. It also remains to be determined whether the examples studied here are typical, or whether they represent peculiar outcomes of our specific choices of parameter values. More generally, the field is open for attempts to characterize the conditions under which the force of natural selection in the presence of recurring deleterious mutation will mold hazard functions into familiar forms.

Acknowledgements This work has been supported by Grant AG-P01-008454 from the U.S. National Institute on Aging. The research of the first author has been supported by the Miller Institute for Basic Research in Science at U.C. Berkeley, and the research of the third author has been supported in part by Grant DMS-0405778 from the U.S. National Science Foundation.

Open Access This article is distributed under the terms of the Creative Commons Attribution Noncommercial License which permits any noncommercial use, distribution, and reproduction in any medium, provided the original author(s) and source are credited.

\section{References}

Bürger, R. (2000). The mathematical theory of selection, recombination, and mutation. Chichester, New York: Wiley.

Campisi, J. (2003). Cellular senescence and apoptosis: How cellular responses might influence aging phenotypes. Experimental Gerontology, 38(1), 5-11.

Carey, J. R. (2003). Longevity: The biology and demography of life span. Princeton: Princeton University Press.

Carey, J. R. \& Tuljapurkar, S. (eds.) (2003). Life span: Evolutionary, ecological, and demographic perspectives. Population Council. Pop. Dev. Rev. vol. 29 suppl.

Charlesworth, B. (1994). Evolution in age-structured populations. Cambridge: Cambridge University Press.

Charlesworth, B. (2000). Fisher, Medawar, Hamilton, and the evolution of aging. Genetics, 156, 927-931.

Charlesworth, B. (2001). Patterns of age-specific means and genetic variances of mortality rates predicted by the mutation-accumulation theory of ageing. Journal of Theoretical Biology, 210(1), 47-65.

Evans, S. N., Steinsaltz, D., \& Wachter, K. W. (2009). A mutation-selection model for general genotypes with recombination. http://arxiv.org/abs/q-bio.PE/0609046.

Finkelstein, M., \& Esaulova, V. (2006). Asymptotic behavior of a general class of mixture failure rates. Advances in Applied Probability, 38(1), 244-262.

Gavrilov, L. A. (1978). A mathematical model of the aging of animals. Doklady Akademii Nauk SSSR, 238, 490-492.

Gavrilov, L. A., \& Gavrilova, N. S. (1991). The biology of lifespan: A quantitative approach Chur, Switzerland: Harwood Academic.

Gavrilov, L. A., \& Gavrilova, N. S. (2001). The reliability theory of aging and longevity. Journal of Theoretical Biology, 213, 527-545.

Haldane, J. B. S. (1937). The effect of variation on fitness. American Naturalist, 71, 337-349.

Hamilton, W. D. (1966). The moulding of senescence by natural selection. Journal of Theoretical Biology, 12, 12-45. 
Hasty, P. (2001). The impact energy metabolism and genome maintenance have on longevity and senescence: Lessons from yeast to mammals. Mechanisms of Ageing and Development, 122(15), 1651-1662.

Hedrick, P. W. (1999). Antagonistic pleiotropy and genetic polymorphism: A perspective. Heredity, 82, 126-1233.

Kallenberg, O. (1983). Random measures. New York: Academic.

Kimura, M., \& Maruyama, T. (1966). The mutational load with epistatic gene interaction. Genetics, $54,1337-1351$.

Medawar, P. (1952). An unsolved problem in biology: An inaugural lecture delivered at University College, London, 6 December, 1951. London: H. K. Lewis.

Nyström, T. (2003). Conditional senescence in bacteria: Death of the immortals. Molecular Microbiology, 48(1), 17-23.

Pearl, R., \& Miner, J. R. (1935). Experimental studies on the duration of life. XIV. The comparative mortality of certain lower organisms. The Quarterly Review of Biology, 10(1) 60-79.

Pletcher, S. D., \& Neuhauser, C. (2000). Biological aging - criteria for modeling and a new mechanistic model. International Journal of Modern Physics C, 11(3), 525-546.

Rose, M. R. (1985). Life history evolution with antagonistic pleiotropy and overlapping generations. Theoretical Population Biology, 28(3), 342.

Rosen, R. (1978). Feedforwards and global system failure: A general mechanism for senescence. Journal of Theoretical Biology, 74, 579-590.

Schnebel, E. M., \& Grossfield, J. (1988). Antagonistic pleiotropy: An interspecific Drosophila comparison. Evolution, 42(2), 306-311.

Steinsaltz, D., Evans, S. N., \& Wachter, K. W. (2005). A generalized model of mutation-selection balance with applications to aging. Advances in Applied Mathematics, 35(1), 16-33.

Strehler, B., \& Mildvan, A. (1960). General theory of mortality and aging. Science, 132(3418), $14-21$.

Toupance, B., Godelle, B., Gouyon, P.H., \& Schächter, F. (1998). A model for antagonistic pleiotropic gene action for mortality and advanced age. The American Journal of Human Genetics, 62(6), 1525-1534.

Vaupel, J. W., Carey, J. R., Christensen, K., Johnson, T. E., Yashin, A. I., Holm, N. V., et al. (1998). Biodemographic trajectories of longevity. Science, 280(5365), 855-860.

Wachter, K. W. (1999). Evolutionary demographic models for mortality plateaus. Proceedings of the National Academy of Sciences, USA, 96, 10544-10547.

Wachter, K. W., Evans, S. N., \& Steinsaltz, D. R. (2008). The age-specific force of natural selection and walls of death. http://arxiv.org/abs/0807.0483.

Wachter, K. W., \& Finch, C. E. (eds.) (1997). Between Zeus and the Salmon: The biodemography of longevity. Washington, D.C.: National Academies.

Weitz, J., \& Fraser, H. (2001). Explaining mortality rate plateaus. Proceedings of the National Academy of Sciences, USA, 98(26), 15383-15386.

Williams, G. C. (1957). Pleiotropy, natural selection, and the evolution of senescence. Evolution, $11,398-411$.

Williams, P. D., \& Day, T. (2003). Antagonistic pleiotropy, mortality source interactions, and the evolutionary theory of senescence. Evolution, 57(7), 1478-1488. 